BONPLANDIA 20(2). 2011

ISSN: 0524-0476

\title{
RECURSOS FITOTERAPÉUTICOS Y COMPORTAMIENTO POBLACIONAL EN DOS SECTORES DE LA RIBERA RIOPLATENSE DE BERISSO, BUENOS AIRES, ARGENTINA
}

\author{
MARCELO P. HERNÁNDEZ1 \& ANA M. ARAMBARRI2, *
}

Summary: Hernández, M. P. \& A. M. Arambarri. 2011. Phytoterapeutic resources and population behavior on the River Plate bank, Berisso, Buenos Aires, Argentina. Bonplandia 20(2): 137-148.

The work discloses of comparative way, the phytotherapy resource used by the inhabitants of two sectors of the Rio de la Plata bank, one continental, ubicated in the area of Palo Blanco and Los Talas localities namely "Monte", and other insular, represented by Paulino Island, both situated northeastern Berisso party, Buenos Aires province, Argentina. The objective is to analyze the behavior of the people in the use of medicinal plants in relation to the environment in which domestic units are located. Results showed that there are differences due to traditional, structural and environmental factors, that lead to greater awareness and use of plants to treat skin diseases, rheumatism, arthritis and asthma in the island, while on the continental zone, is given due prominence to the use of plants to treat parasitic infections and respiratory problems. Of a total of 61 species, is emphasizes the use of Aloe vera (L.) Burm $f$. (Asphodelaceae), Cortaderia selloana (Schult. \& Schult. f.) Asch. \& Graebn. (Poaceae) and Equisetum giganteum L. (Equisetaceae), like the use of one lichen Ramalina celastri (Spreng.) Krog \& Swinscow in the Paulino Island.

Key words: Los Talas, medicinal plants, Palo Blanco, Paulino island.

Resumen: Hernández, M. P. \& A. M. Arambarri. 2011. Recursos fitoterapéuticos y comportamiento poblacional en la ribera rioplatense de Berisso, Buenos Aires, Argentina. Bonplandia 20(2): 137-148.

El trabajo da a conocer de manera comparativa, el recurso fitoterapéutico utilizado por los habitantes de dos sectores de la ribera rioplatense, uno continental, ubicado en las localidades de Palo Blanco y Los Talas denominado "Monte" y, otro insular, representado por la isla Paulino, ambos situados al noreste del partido de Berisso, provincia de Buenos Aires, Argentina. El objetivo es analizar comparativamente el comportamiento de los habitantes en el uso de las plantas medicinales en relación con el ambiente en que se encuentran situadas las unidades domésticas. Los resultados demostraron que existen diferencias atribuibles a factores tradicionales, estructurales y ambientales que conducen a un mayor conocimiento y uso de plantas para tratar afecciones de la piel, reumatismo, artritis y asma en la zona insular, mientras que en la zona continental, se destaca el uso de plantas para tratar parasitosis y problemas de las vías respiratorias. De un total de 61 especies, se destaca el uso de Aloe vera (L.) Burm $f$. (Asphodelaceae), Cortaderia selloana (Schult. \& Schult. f.) Asch. \& Graebn. (Poaceae) y Equisetum giganteum L. (Equisetaceae), así como el uso del liquen Ramalina celastri (Spreng.) Krog \& Swinscow en la Isla Paulino.

Palabras claves: Isla Paulino, Los Talas, plantas medicinales, Palo Blanco.

${ }^{1}$ Sistemática Vegetal, mphciencia@yahoo.com; ${ }^{2}$ Morfología Vegetal, Departamento de Ciencias Biológicas, Facultad de Ciencias Agrarias y Forestales, Universidad Nacional de La Plata, 60 y 119, C.C. 31 (1900) La Plata.

*autor para correspondencia anaramba@yahoo.com.ar 


\section{Introducción}

La zona de estudio comprende un sector continental y otro insular ubicados en el Partido de Berisso, Provincia de Buenos Aires, Argentina. (Fig. 1). Ambos sectores, presentan un clima templado con inviernos benignos, precipitaciones pluviales con una media anual de 800-1.000 mm y ambientes naturales de gran riqueza florística (Lahitte \& al., 1997, 1998) parcialmente modificados por factores antrópicos (i.e., explotaciones agrícola, ganadera, del suelo y asentamiento humano). Actualmente existen distintos tipos de vegetación tales como plantas acuáticas, césped ribereño, matorrales, pajonales, bosques exóticos, bosques nativos relictuales y pequeñas áreas con algunas especies vegetales pertenecientes a una antigua selva marginal que, a fines del siglo XIX y principios del $\mathrm{XX}$, junto a los bosques nativos, se extendía del NE al SE de la provincia de Buenos Aires, desde la localidad de Punta Lara (Partido de Ensenada) hasta la localidad de Magdalena (Partido de Magdalena) (Cabrera, 1949; Arturi, \& al., 2006). En este escenario se encuentran afincados pobladores, algunos de los cuales, desarrollan tareas relacionadas con el cultivo de vid, frutales, flores y apicultura.

En lo que se refiere a estudios y relevamientos sobre plantas medicinales, etnobotánica y etnofarmacobotánica existen numerosas obras y artículos científicos (e. g. Martínez Crovetto, 1964, 1965, 1981; Toursarkissian, 1980; Martínez \& Pochettino, 1992; Lahitte \& Hurrell, 1995-1996; Lahitte \& al., 1997, 1998; Marzocca, 1997; Pochettino \& al., 1997, 2008; Valla \& al., 1999; Arenas 2003; Mandrile, 2003; Alonso \& Desmarchelier, 2005; Barboza \& al., 2006; Keller \& Romero, 2006; Bacigalupo \& al., 2008; Keller, 2008; Hurrell \& al., 2009, 2011; Hilgert \& al., 2010). Estas publicaciones constituyeron la guía para el desarrollo de los estudios previos (Hernández \& al., 2009, 2010) en la zona rioplatense de Berisso, cuyos resultados pusieron de manifiesto la existencia de diferencias en varios aspectos entre los sectores considerados de Palo Blanco-Los Talas (PB-LT) y la Isla Paulino (IP). Esto constituye la base de este nuevo estudio cuyo propósito es analizar comparativamente el comportamiento de sus habitantes en el uso de las plantas medicinales en relación con el ambiente en que se encuentran situados.

\section{Breve reseña histórica del área de estudio}

A fines del siglo XIX y principios del siglo $\mathrm{XX}$, las ciudades de Ensenada, Berisso y zonas cercanas recibieron una gran cantidad de inmigrantes procedentes de diferentes regiones europeas. Las localidades de Palo Blanco y los Talas comenzaron a poblarse entre 1890 y 1910, desarrollándose una intensa actividad hortícola, frutícola y de explotación forestal (Sanucci, 1972). Así mismo, a fines del siglo XIX en la ribera rioplatense existía la "Isla Santiago", perteneciente a los actuales partidos de Berisso y Ensenada. En 1883, cuando se comenzó a construir el Puerto de La Plata, la isla quedó dividida por un canal de acceso a dicho puerto en "Isla Santiago" perteneciente al partido de Ensenada, e "Isla Paulino" perteneciente al partido de Berisso (Fig. 1). Los primeros habitantes de estas islas fueron obreros inmigrantes que trabajaron en la construcción del Puerto y en el dragado de canales de acceso alternativos (Michellod, 2000, 2001). Como consecuencia de la acción antrópica, hoy coexisten en la ribera rioplatense especies vegetales nativas y exóticas que son utilizadas por sus propiedades medicinales. El área de estudio comparativo comprende dos zonas de la ribera platense, la zona costera, inundable, de Palo BlancoLos Talas, donde la población supera los 100 habitantes, quienes además cuentan con todos los servicios y pueden acceder por diversos caminos a las ciudades de La Plata, Berisso y Ensenada y la zona de la Isla Paulino donde la población estable es inferior a 10 familias, que no cuentan con servicios de agua potable, luz, gas ni teléfono, no hay hospital ni sala de emergencias, excepto durante el período estival en que funciona un servicio de enfermería, a esto se suma que la única forma de comunicación es fluvial, existiendo un lanchón comunitario con horario restringido y unas pocas lanchas particulares (Hernández \& al., 2009, 2010). 


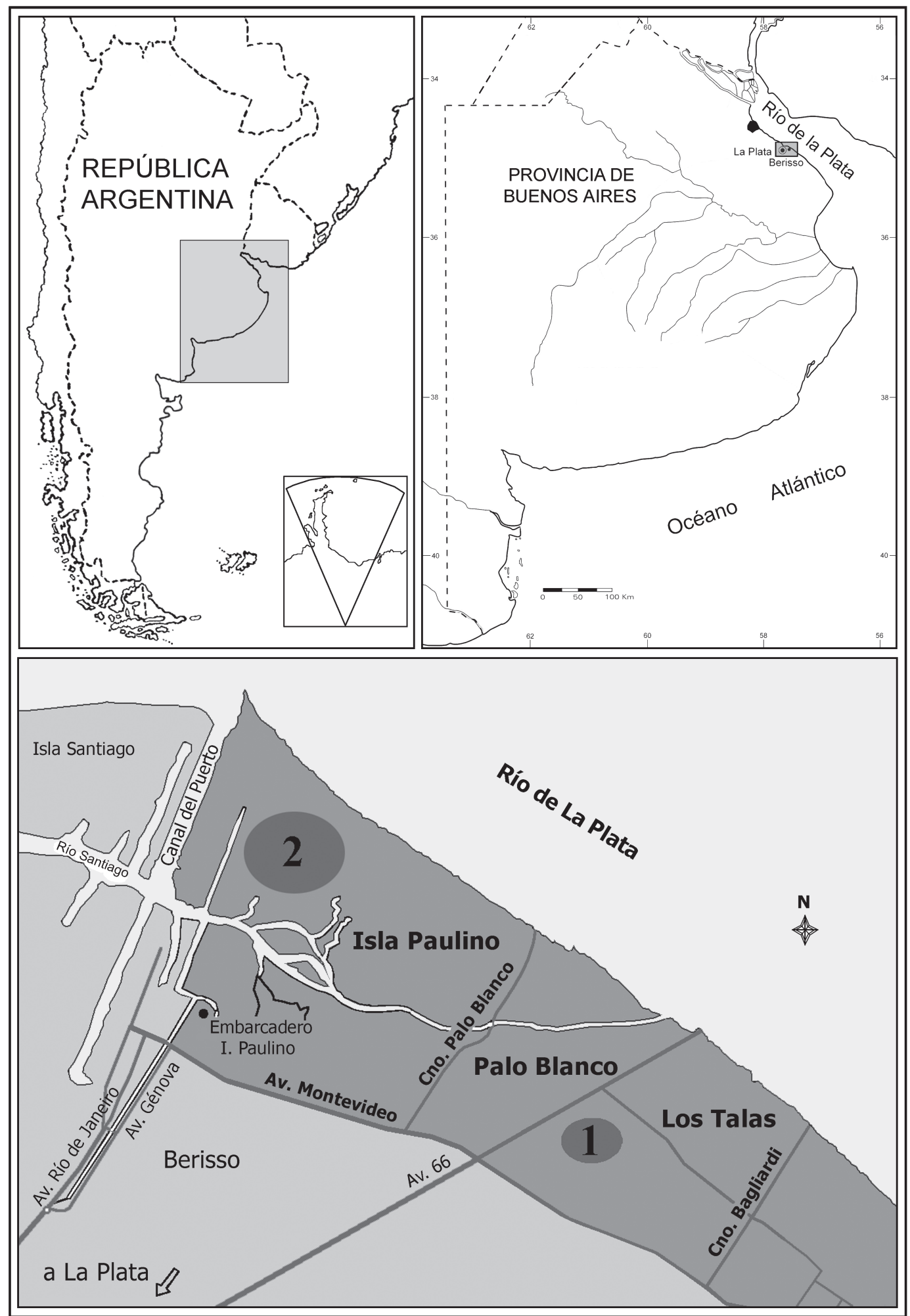

Fig. 1. Ubicación geográfica de la zona de estudio. República Argentina, Provincia de Buenos Aires, Partido de Berisso. 1: zona continental "Palo Blanco-Los Talas"; 2: zona insular "Isla Paulino". 


\section{Material y Métodos}

Se utilizaron los registros previos del relevamiento realizado en las zonas de Palo Blanco-Los Talas (PB-LT) e Isla Paulino (IP), donde se empleó el método etnográfico, con observación de participantes con entrevistas de tipo abiertas, directas e informal-estructuradas de acuerdo con De Paula \& al. (2002). Los datos fueron obtenidos de 50 unidades domésticas (UD) en PB-LT y de 18 UD en IP, esto llevó a encuestar 146 habitantes permanentes en la primera zona y 50 en la isla. Como se indica en los trabajos de relevamiento (Hernández \& al., 2009, 2010), se obtuvo como resultado un listado de 60 especies de plantas vasculares y un liquen empleadas en la medicina folclórica, sumadas ambas regiones. En el presente trabajo se analizaron de manera comparativa los datos de las dos zonas y los resultados se presentan en la tabla 1 y gráficos. En la tabla 1 se indican las especies sus datos botánicosistemáticos, se hace referencia a la planta entera u órgano vegetal utilizado de cada taxón, formas de preparación y administración $\mathrm{y}$ cita el material testigo con su número y las siglas de los herbarios donde se encuentra depositado, herbario del Museo de Ciencias Naturales de La Plata (LP); herbario de la Facultad de Agronomía de La Plata (LPAG) y herbario del Instituto de Botánica Spegazzini de La Plata (LPS). Para la actualización de los nombres científicos de las especies nativas y adventicias se consultaron las obras de Zuloaga \& al. (2008) y el 15-IV-2011 se consultaron los sitios (www2.darwin.edu.ar/) y The International Plant Names Index (www. ipni.org/).

\section{Resultados y Discusión}

El recurso fitoterapéutico utilizado en el área de estudio (PB-LT e IP), está constituido en un $45-48 \%$ de especies nativas y poco más del $50 \%$ por especies exóticas. La mayor utilización de especies introducidas, podría atribuirse a varios factores: la costumbre y conocimiento que tenían los inmigrantes europeos quienes al ingresar a fines del siglo XIX, trajeron sus especies hortícolas, frutales, ornamentales y/o medicinales (Cabrera, 1949); otro factor que podría sumarse al anterior es la proximidad de centros urbanos donde pueden adquirirlas para su reproducción y/o multiplicación frecuentemente como ornamentales, teniendo muchas de ellas una gran capacidad de adaptación y fácil reproducción gámica y agámica, de acuerdo con lo observado por Hernández \& al. (2010) durante el relevamiento de datos. Entre las especies utilizadas, el mayor número pertenece a las familias Asteraceae, Myrtaceae y Verbenaceae, en PB-LT se destaca el uso de dos especies de Aloysia y tres de Verbena (Verbenaceae), mientras en la IP el mayor número corresponde a especies de diversos géneros de la familia Asteraceae (Tabla 1).

Tabla 1. Lista de las plantas vasculares y liquen, empleados en la medicina popular en Palo Blanco-Los Talas (1) e Isla Paulino (2). Se destaca la parte utilizada, forma de preparación y de administración, porcentaje de unidades domésticas (UD) en donde se usa cada especie y la cita del material testigo. Leg. A: Amorín ; De: Delucchi, G.; H: Hernández, M.P.; HJ: Hurrell, J.; HP: Haloua, P.; OH: Otero Hernández; P: Pochettino \& Pertusi; R: Rosato, V.G.; S: Scala, A.; TR: Torres Robles.

\begin{tabular}{lllll}
\hline \multicolumn{1}{c}{ Plantas Vasculares } & \multicolumn{1}{c}{$\begin{array}{c}\text { Nombres } \\
\text { vulgares }\end{array}$} & $\begin{array}{c}\text { Parte utilizada/ } \\
\text { Preparación/ } \\
\text { Administración }\end{array}$ & UD (\%) & Testigo \\
\hline $\begin{array}{l}\text { ALLIACEAE } \\
\text { Allium cepa } \text { L. }\end{array}$ & Cebolla & Catáfila/Infusión/Oral & $2,5 \%(1)$ & $\begin{array}{c}\text { De } 1049 \\
\text { (LP) }\end{array}$ \\
$\begin{array}{l}\text { AMARYLLIDACEAE } \\
\text { Narcissus tazetta } \text { L. }\end{array}$ & Junquillo blanco & $\begin{array}{l}\text { Hoja y flor/ } \\
\text { Infusión/Oral }\end{array}$ & $2,5 \%(1)$ & De 751 (LPAG) \\
\hline
\end{tabular}




\begin{tabular}{|c|c|c|c|c|}
\hline Plantas Vasculares & $\begin{array}{l}\text { Nombres } \\
\text { vulgares }\end{array}$ & $\begin{array}{l}\text { Parte utilizada/ } \\
\text { Preparación/ } \\
\text { Administración }\end{array}$ & UD (\%) & Testigo \\
\hline \multicolumn{5}{|l|}{ ANACARDIACEAE } \\
\hline Schinus areira $\mathrm{L}$. & Molle, aguaribay & $\begin{array}{l}\text { Hoja/Infusión/ } \\
\text { Oral }\end{array}$ & $5,5 \%(2)$ & $\begin{array}{l}\text { H } 3 \\
\text { (LPAG) }\end{array}$ \\
\hline \multicolumn{5}{|l|}{ ARALIACEAE } \\
\hline $\begin{array}{l}\text { Tetrapanax papyrifera (Hook.) } \\
\text { K. Koch. }\end{array}$ & Árvore & $\begin{array}{l}\text { Hoja/Infusión/ } \\
\text { Oral }(1,2)\end{array}$ & $\begin{array}{l}2,5 \%(1) ; 5,5 \% \\
(2)\end{array}$ & H 22 (LPAG) \\
\hline \multicolumn{5}{|l|}{ ASPHODELACEAE } \\
\hline Aloe arborescens Mill. & Aloe & $\begin{array}{l}\text { Hoja fresca/ } \\
\text { Tópico }(1,2)\end{array}$ & $\begin{array}{l}5 \%(1) \\
5,5 \%(2)\end{array}$ & H 39 (LPAG) \\
\hline Aloe vera (L.) Burm. $f$. & Aloe & $\begin{array}{l}\text { Hoja fresca/ } \\
\text { Tópico (1); Tintura/ } \\
\text { Tópico (2) }\end{array}$ & $\begin{array}{l}5 \%(1) \\
44,4 \%(2)\end{array}$ & H 29 (LPAG) \\
\hline \multicolumn{5}{|l|}{ ASTERACEAE } \\
\hline $\begin{array}{l}\text { Achyrocline satureioides } \\
\text { (Lam.) DC. }\end{array}$ & $\begin{array}{l}\text { Marcela, } \\
\text { marcelita (1) }\end{array}$ & $\begin{array}{l}\text { Hoja/Infusión/ } \\
\text { Oral (1) }\end{array}$ & $2,5 \%(1)$ & $\begin{array}{l}\text { HP } 3543 \\
\text { (LP) }\end{array}$ \\
\hline Baccharis trimera (Less.) DC. & Carqueja & $\begin{array}{l}\text { Tallo y hoja/ } \\
\text { Decocción e infusión/ } \\
\text { Oral }\end{array}$ & $22,2 \%(2)$ & H 34 (LPAG) \\
\hline Artemisia absinthium $\mathrm{L}$. & Ajenjo & $\begin{array}{l}\text { Hoja/Infusión/ } \\
\text { Oral }\end{array}$ & $5 \%(1)$ & $\begin{array}{l}\text { TR } 2639 \\
\text { (LP) }\end{array}$ \\
\hline Matricaria recutita $\mathrm{L}$. & Manzanilla & $\begin{array}{l}\text { Flor/Infusión/ } \\
\text { Oral }(1,2)\end{array}$ & $\begin{array}{l}15 \%(1) \\
5,5 \%(2)\end{array}$ & $\begin{array}{l}\text { H } 12 \\
\text { (LPAG) }\end{array}$ \\
\hline Solidago chilensis Meyen & Vara de oro & $\begin{array}{l}\text { Hoja/Infusión/ } \\
\text { Oral }\end{array}$ & $5,5 \%(2)$ & H 17 (LPAG) \\
\hline $\begin{array}{l}\text { Taraxacum officinale G. Weber } \\
\text { ex F.H. Wigg. }\end{array}$ & Diente de león & $\begin{array}{l}\text { Hoja y raíz/ } \\
\text { Decocción e Infusión/ } \\
\text { Oral }\end{array}$ & $5,5 \%(2)$ & H 27 (LPAG) \\
\hline Xanthium spinosum $\mathrm{L}$. & Abrojo & $\begin{array}{l}\text { Hoja/Decocción e } \\
\text { infusión/Oral (1); Hoja } \\
\text { fresca/ } \\
\text { Decocción/ Tópico (2) }\end{array}$ & $\begin{array}{l}2,5 \%(1) ; 5,5 \% \\
(2)\end{array}$ & H 38 (LPAG) \\
\hline \multicolumn{5}{|l|}{ BRASSICACEAE } \\
\hline Lepidium aletes J. F. Macbr. & Mastuerzo & $\begin{array}{l}\text { Hoja/Decocción e } \\
\text { Infusión/Oral }\end{array}$ & $5 \%(1)$ & $\begin{array}{l}\text { Da 778; TR } 541 \\
\text { (LP) }\end{array}$ \\
\hline \multicolumn{5}{|l|}{ CANNACEAE } \\
\hline Canna indica $\mathrm{L}$. & Achira & $\begin{array}{l}\text { Rizoma/ } \\
\text { Decocción/ } \\
\text { Oral }\end{array}$ & $5,5 \%(2)$ & H 23 (LPAG) \\
\hline \multicolumn{5}{|l|}{ CELTIDACEAE } \\
\hline $\begin{array}{l}\text { Celtis ehrenbergiana } \\
\text { (Klotzsch) Liebm. } \\
\text { CHENOPODIACEAE }\end{array}$ & Tala & $\begin{array}{l}\text { Hoja/Infusión/ } \\
\text { Oral }\end{array}$ & $7,5 \%(1)$ & H 42 (LPAG) \\
\hline Chenopodium ambrosioides L. & Paico macho & $\begin{array}{l}\text { Hoja/Infusión/ } \\
\text { Oral }(1,2)\end{array}$ & $\begin{array}{l}7,5 \%(1) ; 5,5 \% \\
(2)\end{array}$ & H 10 (LPAG) \\
\hline $\begin{array}{l}\text { Chenopodium retusum (Juss.) } \\
\text { ex Moq. } \\
\text { COMMELINACEAE }\end{array}$ & Paico & $\begin{array}{l}\text { Hoja/Infusión/ } \\
\text { Oral }\end{array}$ & $7,5 \%(1)$ & H 2401 (LP) \\
\hline Commelina diffusa Burm. $f$. & $\begin{array}{l}\text { Flor de Santa } \\
\text { Lucía }\end{array}$ & $\begin{array}{l}\text { Hojas frescas/ } \\
\text { Tópico }\end{array}$ & $5,5 \%(2)$ & H 36 (LPAG) \\
\hline \multicolumn{5}{|l|}{ EQUISETACEAE } \\
\hline Equisetum giganteum L. & Cola de caballo & $\begin{array}{l}\text { Hoja, Planta entera/ } \\
\text { Decocción/Oral }(1,2)\end{array}$ & $\begin{array}{l}45 \%(1) \\
44,4 \%(2)\end{array}$ & H 21 (LPAG) \\
\hline
\end{tabular}




\begin{tabular}{|c|c|c|c|c|}
\hline Plantas Vasculares & $\begin{array}{l}\text { Nombres } \\
\text { vulgares }\end{array}$ & $\begin{array}{l}\text { Parte utilizada/ } \\
\text { Preparación/ } \\
\text { Administración }\end{array}$ & UD (\%) & Testigo \\
\hline \multicolumn{5}{|l|}{ EUPHORBIACEAE } \\
\hline Euphorbia serpens Kunth & Yerba meona & $\begin{array}{l}\text { Planta entera/ } \\
\text { Infusión/Oral }\end{array}$ & $5,5 \%(2)$ & H11 (LPAG) \\
\hline \multicolumn{5}{|l|}{ FABACEAE } \\
\hline $\begin{array}{l}\text { Bauhinia forficata Link subsp. } \\
\text { pruinosa (Vogel) Fortunato \& } \\
\text { Wunderlin }\end{array}$ & Pata de vaca & $\begin{array}{l}\text { Hoja/Infusión/ } \\
\text { Oral }(1,2)\end{array}$ & $\begin{array}{l}5 \%(1) \\
11,1 \%(2)\end{array}$ & H 1 (LPAG) \\
\hline \multicolumn{5}{|l|}{ IRIDACEAE } \\
\hline Iris pseudacorus L. & Lirio amarillo & $\begin{array}{l}\text { Rizoma frescos/ } \\
\text { Infusión, en polvo/Oral } \\
\text { e Inhalación }\end{array}$ & $5 \%(1)$ & OH 1264 (LP) \\
\hline \multicolumn{5}{|l|}{ JUGLANDACEAE } \\
\hline $\begin{array}{l}\text { Carya illinoiensis (Wangenh.) } \\
\text { K. Koch. }\end{array}$ & Nuez de Pecán & $\begin{array}{l}\text { Exocarpo/Tintura con } \\
\text { grapa/Oral }\end{array}$ & $5,5 \%(2)$ & H 7 (LPAG) \\
\hline \multicolumn{5}{|l|}{ LAMIACEAE } \\
\hline Mentha $x$ piperita $\mathrm{L}$. & $\begin{array}{l}\text { Menta, menta } \\
\text { piperita }\end{array}$ & $\begin{array}{l}\text { Hoja/Infusión/ } \\
\text { Oral }(1,2)\end{array}$ & $\begin{array}{l}15 \%(1) \\
5,5 \%(2)\end{array}$ & H 13 (LPAG) \\
\hline Origanum vulgare $\mathrm{L}$. & Orégano & $\begin{array}{l}\text { Hoja/Infusión/ } \\
\text { Oral }\end{array}$ & $11,1 \%(2)$ & H 2 (LPAG) \\
\hline Rosmarinus officinalis $\mathrm{L}$. & Romero & $\begin{array}{l}\text { Hoja/Infusión/ } \\
\text { Oral (1); Hoja/Tintura/ } \\
\text { Tópico (2) }\end{array}$ & $\begin{array}{l}7,5 \%(1) ; 5,5 \% \\
(2)\end{array}$ & H 37 (LPAG) \\
\hline Salvia officinalis L. & Salvia & $\begin{array}{l}\text { Hoja/Infusión/ } \\
\text { Oral }\end{array}$ & $\begin{array}{l}2,5 \%(1) ; 5,5 \% \\
(2)\end{array}$ & $\begin{array}{l}\mathrm{H} 4 \\
\text { (LPAG) }\end{array}$ \\
\hline \multicolumn{5}{|l|}{ LAURACEAE } \\
\hline Laurus nobilis L. & Laurel & $\begin{array}{l}\text { Hoja/Infusión/ } \\
\text { Oral }(1,2)\end{array}$ & $\begin{array}{l}15 \%(1) \\
5,5 \%(2)\end{array}$ & H 20 (LPAG) \\
\hline \multicolumn{5}{|l|}{ MALVACEAE } \\
\hline Sida rhombifolia $\mathrm{L}$. & Escoba dura & $\begin{array}{l}\text { Hoja/Infusión/ } \\
\text { Oral }\end{array}$ & $5,5 \%(2)$ & H 18 (LPAG) \\
\hline $\begin{array}{l}\text { Sphaeralcea bonariensis (Cav.) } \\
\text { Griseb. }\end{array}$ & $\begin{array}{l}\text { Malva, } \\
\text { malvavisco }\end{array}$ & $\begin{array}{l}\text { Hoja/Decocción/ } \\
\text { Oral }\end{array}$ & $2,5 \%(1)$ & $\begin{array}{l}\text { HJ } 6868 \\
\text { (LP) }\end{array}$ \\
\hline \multicolumn{5}{|c|}{ MELIACEAE } \\
\hline Melia azedarach L. & Paraíso & $\begin{array}{l}\text { Hoja/Decocción/ } \\
\text { Tópico }\end{array}$ & $5,5 \%(2)$ & $\begin{array}{l}\text { H } 8 \\
\text { (LPAG) }\end{array}$ \\
\hline \multicolumn{5}{|l|}{ MORACEAE } \\
\hline Morus alba $\mathrm{L}$. & $\begin{array}{l}\text { Mora, morera } \\
\text { blanca }\end{array}$ & $\begin{array}{l}\text { Hoja/Decocción e } \\
\text { Infusión/Oral }\end{array}$ & $5 \%(1)$ & $\begin{array}{l}\text { De } 2554 \\
\text { (LP) }\end{array}$ \\
\hline \multicolumn{5}{|l|}{ MUSACEAE } \\
\hline Musa x paradisiaca $\mathrm{L}$. & Plátano, banano & $\begin{array}{l}\text { Hoja fresca/ } \\
\text { Tópico }\end{array}$ & $5,5 \%(2)$ & H 24 (LPAG) \\
\hline \multicolumn{5}{|l|}{ MYRTACEAE } \\
\hline $\begin{array}{l}\text { Blepharocalyx salicifolius } \\
\text { (Kunth) O. Berg }\end{array}$ & $\begin{array}{l}\text { Anacahuita, } \\
\text { arrayán }\end{array}$ & Hoja/Decocción/Oral & $15 \%(1)$ & H 43 (LPAG) \\
\hline $\begin{array}{l}\text { Eucalyptus camaldulensis } \\
\text { Dehnh. }\end{array}$ & Eucalipto & $\begin{array}{l}\text { Hoja y fruto/ } \\
\text { Decocción/ } \\
\text { Inhalación }\end{array}$ & $5,5 \%(2)$ & H 16 (LPAG) \\
\hline $\begin{array}{l}\text { Eucalyptus cinerea F. Müll. ex } \\
\text { Benth. }\end{array}$ & $\begin{array}{l}\text { Eucalipto } \\
\text { plateado }\end{array}$ & $\begin{array}{l}\text { Hoja y fruto/ } \\
\text { Decocción/Inhalación } \\
\text { y Oral }\end{array}$ & $5,5 \%(2)$ & H 35 (LPAG) \\
\hline
\end{tabular}




\begin{tabular}{|c|c|c|c|c|}
\hline Plantas Vasculares & $\begin{array}{l}\text { Nombres } \\
\text { vulgares }\end{array}$ & $\begin{array}{l}\text { Parte utilizada/ } \\
\text { Preparación/ } \\
\text { Administración }\end{array}$ & UD (\%) & Testigo \\
\hline Eucalyptus globulus Labill. & Eucalipto & $\begin{array}{l}\text { Hoja y fruto/ } \\
\text { Vapor/Inhalación }\end{array}$ & $15 \%(1)$ & P 34 (LPAG) \\
\hline Eugenia uniflora $\mathrm{L}$. & Ñangapirí & $\begin{array}{l}\text { Hoja/Infusión/ } \\
\text { Oral }\end{array}$ & $5,5 \%(2)$ & $\begin{array}{l}\text { H } 9 \\
\text { (LPAG) }\end{array}$ \\
\hline \multicolumn{5}{|l|}{ PASSIFLORACEAE } \\
\hline Passiflora caerulea L. & Pasionaria & $\begin{array}{l}\text { Hoja/Infusión/ } \\
\text { Oral }\end{array}$ & $5,5 \%(2)$ & H 25 (LPAG) \\
\hline \multicolumn{5}{|l|}{ PHYLLANTHACEAE } \\
\hline Phyllanthus niruri L. & Rompepiedras & $\begin{array}{l}\text { Hoja/Decocción/ } \\
\text { Oral }\end{array}$ & $2,5 \%(1)$ & $\begin{array}{l}\text { HP } 2838 \\
\text { (LP) }\end{array}$ \\
\hline $\begin{array}{l}\text { Phyllanthus sellowianus } \\
\text { (Klotzch) Müll. Arg. }\end{array}$ & Sarandí blanco & $\begin{array}{l}\text { Hoja/Decocción/ } \\
\text { Oral (1); Hoja y tallo/ } \\
\text { Infusión/ Oral ( } 2 \text { ) }\end{array}$ & $\begin{array}{l}2,5 \%(1) \\
11,1 \%(2)\end{array}$ & $\begin{array}{l}\text { H } 6 \\
\text { (LPAG) }\end{array}$ \\
\hline \multicolumn{5}{|l|}{ PLANTAGINACEAE } \\
\hline Plantago major L. & Llantén & $\begin{array}{l}\text { Hoja fresca/ } \\
\text { Infusión/Tópico }\end{array}$ & $11,1 \%(2)$ & H 26 (LPAG) \\
\hline \multicolumn{5}{|l|}{ POACEAE } \\
\hline Arundo donax L. & Caña de castilla & $\begin{array}{l}\text { Rizoma/ } \\
\text { Decocción/ } \\
\text { Oral }\end{array}$ & $5 \%(1)$ & H 41 (LPAG) \\
\hline $\begin{array}{l}\text { Cortaderia selloana (Schult. \& } \\
\text { Schult. f.) Asch. \& Graebn. }\end{array}$ & Cola de zorro & $\begin{array}{l}\text { Hoja/Decocción/Oral } \\
\text { (1)/Infusión/Oral (2) }\end{array}$ & $\begin{array}{l}50 \%(1) \\
5,5 \%(2)\end{array}$ & H 31 (LPAG) \\
\hline Cymbopogon citratus Stapf & Pasto limón & $\begin{array}{l}\text { Hoja/Infusión/ } \\
\text { Oral }\end{array}$ & $5,5 \%(2)$ & H 30 (LPAG) \\
\hline \multicolumn{5}{|l|}{ POLYGONACEAE } \\
\hline $\begin{array}{l}\text { Muehlenbeckia sagittifolia } \\
\text { (Ortega) Meissn. }\end{array}$ & $\begin{array}{l}\text { Zarza parrilla } \\
\text { colorada }\end{array}$ & $\begin{array}{l}\text { Tallo y raíz/ } \\
\text { Decocción/ Inhalación } \\
\text { y Oral }\end{array}$ & $11,1 \%(2)$ & H 14 (LPAG) \\
\hline \multicolumn{5}{|l|}{ RANUNCULACEAE } \\
\hline Ranunculus muricatus L. & Pata de gallo (1) & $\begin{array}{l}\text { Hoja/Decocción/ } \\
\text { Oral (1) }\end{array}$ & $2,5 \%(1)$ & $\begin{array}{l}\text { De } 2215 \\
\text { (LP) }\end{array}$ \\
\hline \multicolumn{5}{|l|}{ ROSACEAE } \\
\hline Rosa banksiae Ait. & Rosa mosqueta & $\begin{array}{l}\text { Flor/Decocción e } \\
\text { infusión/Oral }\end{array}$ & $2,5 \%(1)$ & A (LPAG 1695) \\
\hline \multicolumn{5}{|l|}{ RUTACEAE } \\
\hline Ruta chalepensis L. & Ruda & $\begin{array}{l}\text { Hoja y tallo/Decocción } \\
\text { e Infusión/Oral }\end{array}$ & $7,5 \%(1)$ & H 44 (LPAG) \\
\hline \multicolumn{5}{|l|}{ SALICACEAE } \\
\hline Salix babylonica L. & Sauce & $\begin{array}{l}\text { Hoja/Infusión/ } \\
\text { Oral }\end{array}$ & $5,5 \%(2)$ & H 32 (LPAG) \\
\hline Salix humboldtiana Willd. & Sauce criollo & $\begin{array}{l}\text { Hoja/Infusión/ } \\
\text { Oral }\end{array}$ & $5,5 \%(2)$ & H 5 (LPAG) \\
\hline \multicolumn{5}{|l|}{ SOLANACEAE } \\
\hline Nicotiana glauca Graham & Palán-palán & $\begin{array}{l}\text { Hoja fresca/ } \\
\text { Decocción/ } \\
\text { Tópico }\end{array}$ & $11,1 \%(2)$ & H 40 (LPAG) \\
\hline $\begin{array}{l}\text { TILIACEAE } \\
\text { Tilia viridis (Bayer) L. } \\
\text { Simonkai nothosubsp. moltkei } \\
\text { (Dippel) Xifreda }\end{array}$ & Tilo & $\begin{array}{l}\text { Flor/Infusión/ } \\
\text { Oral }\end{array}$ & $5,5 \%(2)$ & H 28 (LPAG) \\
\hline
\end{tabular}


BONPLANDIA 20(2). 2011

\begin{tabular}{|c|c|c|c|c|}
\hline Plantas Vasculares & $\begin{array}{c}\text { Nombres } \\
\text { vulgares }\end{array}$ & $\begin{array}{l}\text { Parte utilizada/ } \\
\text { Preparación/ } \\
\text { Administración }\end{array}$ & UD (\%) & Testigo \\
\hline \multicolumn{5}{|l|}{ VERBENACEAE } \\
\hline Aloysia citriodora Palau & Cedrón & $\begin{array}{l}\text { Hoja/Infusión/ } \\
\text { Oral }(1,2)\end{array}$ & $\begin{array}{l}15 \%(1) \\
16,7 \%(2)\end{array}$ & H 33 (LPAG) \\
\hline $\begin{array}{l}\text { Aloysia polystachya (Griseb.) } \\
\text { Moldenke }\end{array}$ & $\begin{array}{l}\text { Burrito, burro, } \\
\text { cedrón, poleo }\end{array}$ & $\begin{array}{l}\text { Hoja y tallo/ } \\
\text { Decocción e Infusión/ } \\
\text { Oral (1); } \\
\text { Hoja/Infusión/ } \\
\text { Oral (2) }\end{array}$ & $\begin{array}{l}15 \%(1) ; 11,1 \% \\
(2)\end{array}$ & H 15 (LPAG) \\
\hline Lantana camara $\mathrm{L}$. & Lantana, salvia & $\begin{array}{l}\text { Flor/Infusión/ } \\
\text { Oral }\end{array}$ & $11,1 \%(2)$ & H 19 (LPAG) \\
\hline Verbena bonariensis L. & Verbena & $\begin{array}{l}\text { Hoja/Infusión/ } \\
\text { Oral }\end{array}$ & $2,5 \%(1)$ & TR 7138 (LP) \\
\hline Verbena intermedia DC. & Verbena & $\begin{array}{l}\text { Hoja/Infusión/ } \\
\text { Oral }\end{array}$ & $2,5 \%(1)$ & TR 7139 (LP) \\
\hline Verbena rigida Spreng. & Verbena & $\begin{array}{l}\text { Hoja/Infusión/ } \\
\text { Oral }\end{array}$ & $2,5 \%(1)$ & S 5621 (LP) \\
\hline \multicolumn{5}{|l|}{ Liquen- RAMALINACEAE } \\
\hline $\begin{array}{l}\text { Ramalina celastri (Spreng.) } \\
\text { Krog \& Swinscow }\end{array}$ & No se conoce & $\begin{array}{l}\text { Organismo entero/ } \\
\text { Infusión/Gargarismo }\end{array}$ & $5,5 \%(2)$ & R (LPS 48.255) \\
\hline
\end{tabular}

En ambas zonas, continental e insular, el órgano vegetal más utilizado es la hoja, el principal uso para problemas digestivos y en la IP para enfermedades de la piel. La forma de preparación característica es la infusión administrada por vía oral. Esto se debe a que los habitantes de la región consideran que la infusión de hojas como bebida, constituye un remedio casero de fácil y rápida elaboración $y$, de gran efectividad. La segunda forma de administración más frecuente es como tópico en la IP (Tabla 1 y Gráficos 1-4).

En ambos sectores, los habitantes utilizan el recurso vegetal para el tratamiento de trastornos de la piel, del aparato digestivo, respiratorio, urinario, como anticefalálgico, antidiabético, cardiotónico, depurativo y sedativo (Hernández \& al., 2009, 2010). Los habitantes de ambas zonas utilizan doce especies vegetales contra afecciones de la piel, dos de ellas son las mismas (Aloe arborescens y $A$. vera). En este sentido, en PB-LT utilizan las plantas para el tratamiento de heridas cortantes, inflamación de las zonas afectadas, en cosmética contra el exceso de grasa superficial y, como agente suavizante. A diferencia del sector continental, los habitantes de la isla Paulino utilizan este recurso para el tratamiento de picaduras de insectos y quemaduras por exposición excesiva al sol e inflamación de las zonas afectadas (Gráfico 2). Hernández \& al. (2010) concluyeron que los últimos usos en la isla, se deberían a dos factores: por un lado a una extensión del período estival y, por otra parte al difícil acceso a las ciudades cercanas que los lleva a tener un mayor conocimiento del efecto de algunas plantas contra tales trastornos y preparar sus propios medicamentos vegetales. Los habitantes del área estudiada (continental e insular) utilizan veinticinco especies vegetales para combatir los trastornos del aparato digestivo y nueve para trastornos del aparato respiratorio. Las afecciones respiratorias, así como las reumáticas y artríticas, que en la zona insular tratan con Aloe arborescens y Rosmarinus officinalis, afectan la población durante el invierno, cuando se combinan las condiciones ambientales de frío y alta humedad, ya que se producen inundaciones y/o anegamientos causados por el ciclo de mareas del río de La Plata y por el fenómeno climático de las tormentas conocidas como "sudestadas" que azotan con frecuencia la región. En ambas zonas, continental e insular, también se usan especies vegetales para trastornos en el aparato 
Gráfico 1. Nos muestra la proporción en que la planta o el órgano vegetal es utilizado. En ambas regiones es la hoja, en menor proporción se encuentran el tallo, la flor y el fruto. En IP se suma la utilización de la planta entera y raíces.

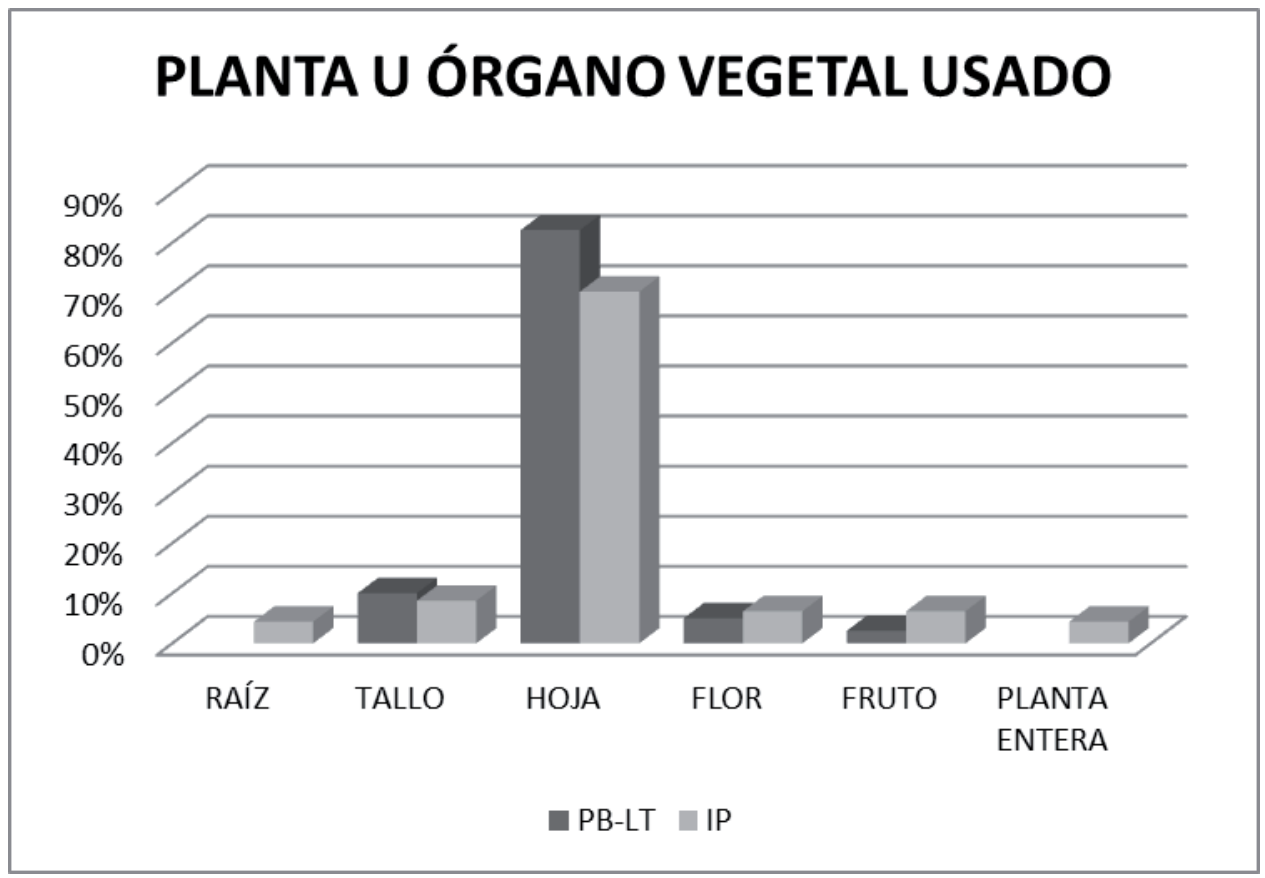

Gráfico 2. Nos diferencia la utilización de las plantas vasculares en la zona continental y la zona insular. En ambas regiones el principal uso es en problemas digestivos. Sin embargo, mientras en PB-LT se utilizan principalmente en problemas del aparato digestivo y sistema nervioso, en IP se usan en alto porcentaje medicinas que actúan en problemas de la piel.

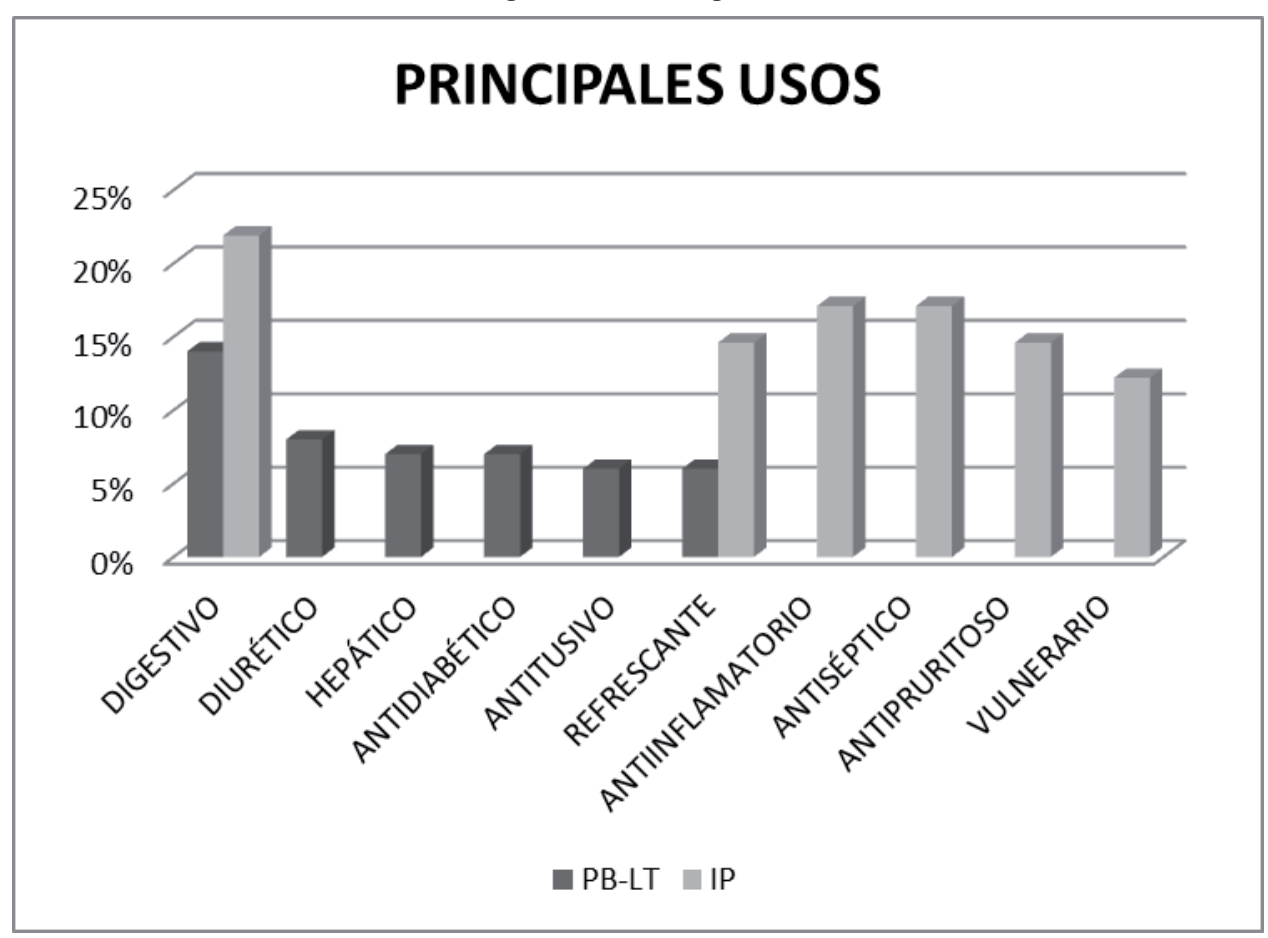


Gráfico 3. Las formas más frecuentes de preparación de los medicamentos en ambas regiones son la infusión y decocción. En IP se destaca también el uso de hojas frescas y tintura en relación con los problemas de la piel que aquejan a los habitantes de la isla.

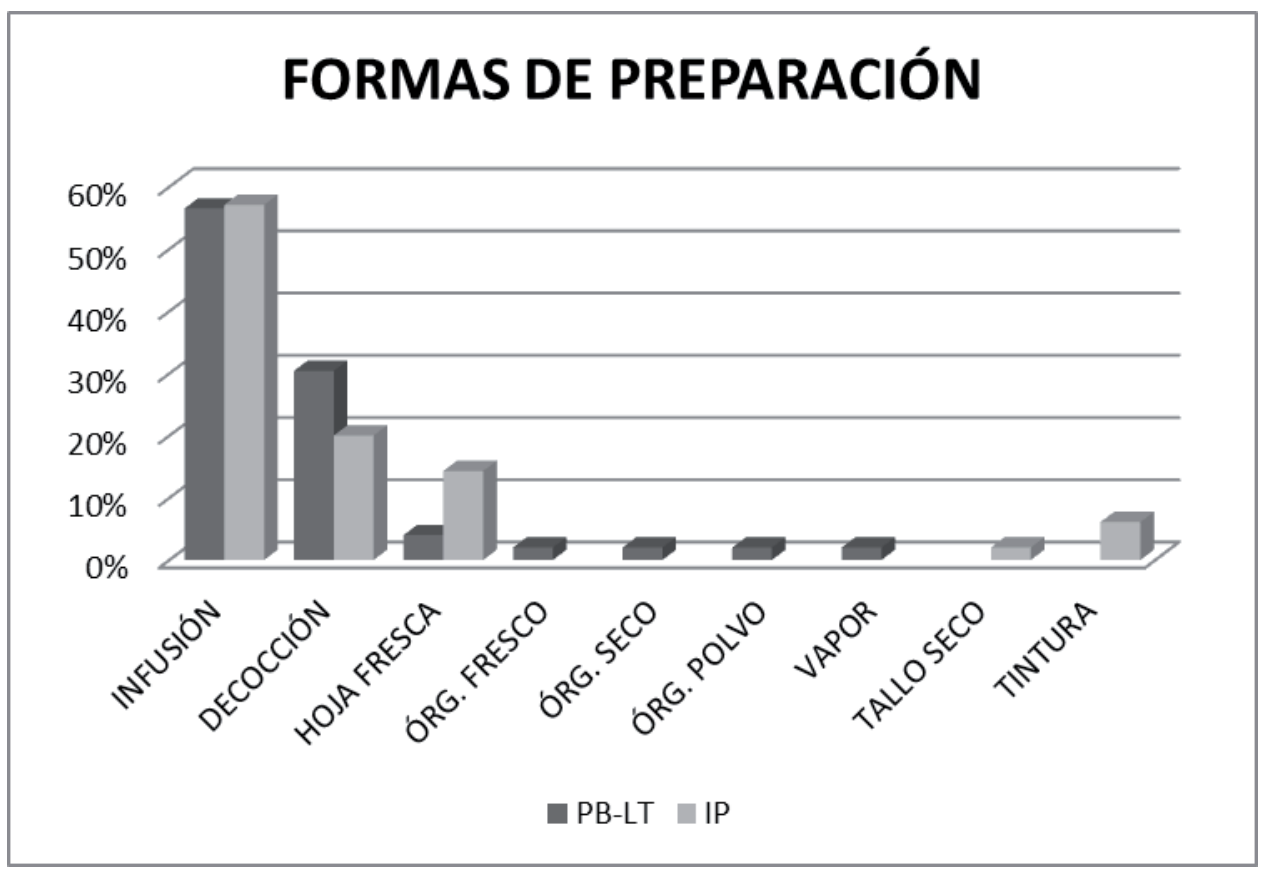

Gráfico 4. La forma de administración más frecuente es oral, en ambas regiones. En IP también es importante la administración como tópico.

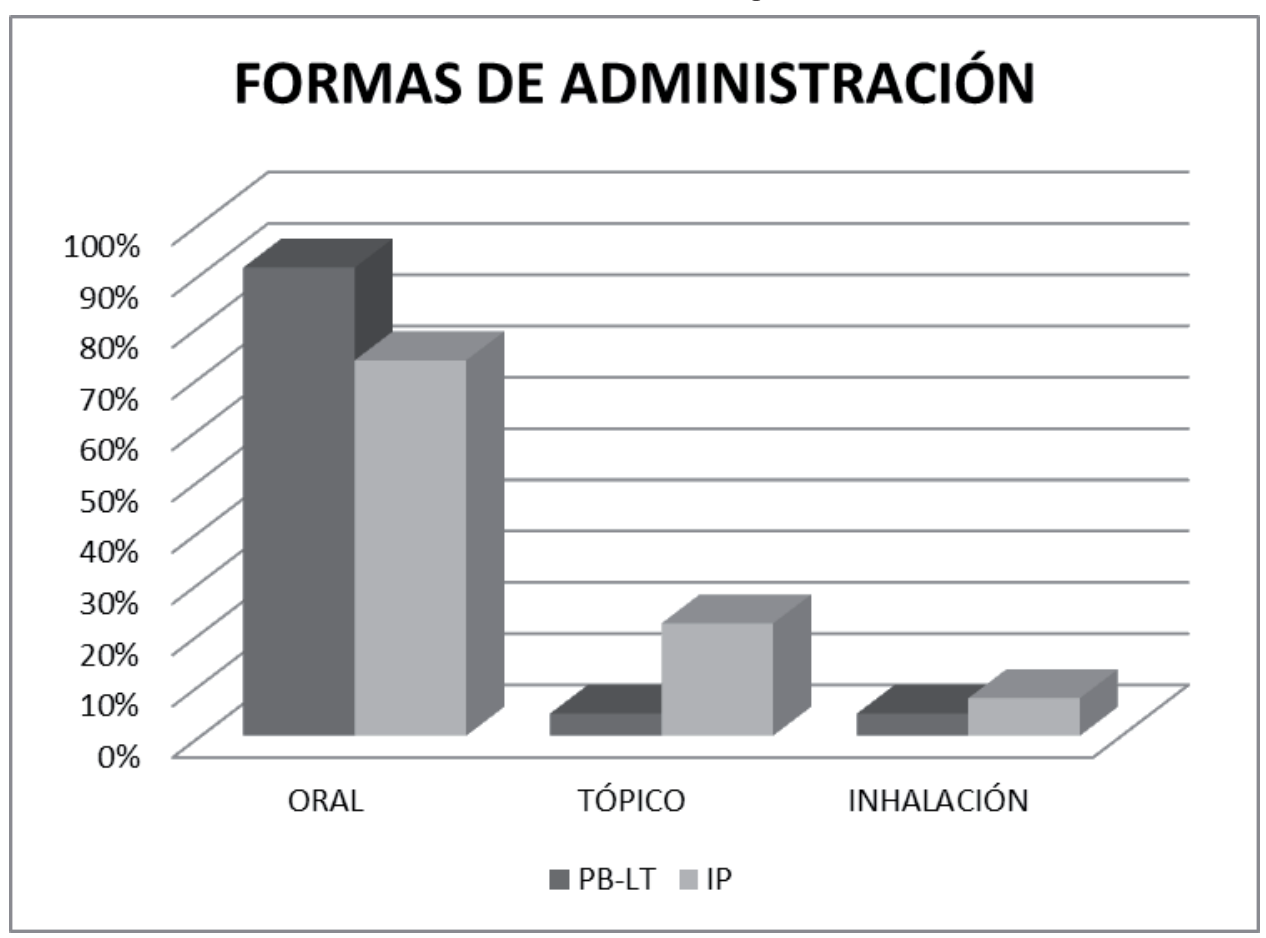


urinario (e. g. cistitis), Celtis ehrenbergiana, Salix spp. y Solidago chilensis son usadas como anticefalálgico; como antidiabético se destaca el uso de Bauhinia forficata subsp. pruinosa y Phyllanthus sellowianus. Para la eliminación de toxinas de la sangre se utilizan tres especies (Morus alba, Rosa banksiae y Xanthium spinosum) en PBLT y cuatro diferentes (Bauhinia forficata subsp. pruinosa, Muehlenbeckia sagittifolia, Phyllantus sellowianus y Taraxacum officinale) en IP. Cabe destacar que en la isla Paulino, también se utiliza como agente antiséptico una especie liquénica (Ramalina celastri) en gargarismos para combatir la infección de garganta. Este análisis coincide con Hernández \& al. (2010) en la existencia de un mayor número de especies usadas en la región insular y de un mayor conocimiento de las propiedades y uso de las plantas medicinales, lo cual estaría relacionado al aislamiento de los isleños, dada la dificultad de traslado a las ciudades cercanas para adquirir productos en farmacias. En relación con esto, también es mayor el número de afecciones tratadas en la isla, este valor elevado es más significativo cuando se considera el bajo número de unidades domésticas con que se trabajó como consecuencia de la escasa población permanente en la isla. Se reporta el uso generalizado de Cortaderia selloana y Equisetum giganteum en el sector continental $\mathrm{y}$, de Aloe vera y Equisetum giganteum en el sector insular, lo cual, como lo expresaran Hernández \& al. (2009, 2010), estaría relacionado con la abundancia y disponibilidad anual de estas especies en la zona, la facilidad para su identificación por las características morfológicas, así como su permanencia a lo largo del año y, al conocimiento ampliamente difundido de sus efectos terapéuticos, tanto en el área de estudio como fuera de ésta. En resumen, existen notables diferencias entre las dos regiones estudiadas atribuibles a factores históricos, estructurales y del ambiente.

\section{Agradecimientos}

A los habitantes de Palo Blanco-Los Talas e Isla Paulino por su colaboración brindando datos de su conocimiento sobre las plantas utilizadas. A la Profesora María Alejandra Migoya por la confección del mapa, a los Curadores de los herbarios y a los revisores anónimos por las sugerencias brindadas que contribuyeron a mejorar el manuscrito.

\section{Bibliografía}

ALONSO, J. \& C. DESMARCHELIER. 2005. Plantas medicinales autóctonas de la Argentina. Bases científicas para su aplicación en atención primaria de la salud, Ed. L.O.L.A. (Literature of Latin America), Buenos Aires. 663 pp.

ARENAS, P. 2003. Etnografía y alimentación entre los Toba-Nachilamole'ek y Wichi-Lhuku'tas del Chaco central (Argentina). Latin Gráfica S.R.L., Buenos Aires. 562 pp.

ARTURI, M. F.; C. A. PÉREZ; M. HORLENT; J. F. GOYA \& S. TORRES ROBLES. 2006. El manejo de los talares de Magdalena y Punta Indio como estrategia para su conservación. En: Mérida E. \& J. Athor (eds.), Talares bonaerenses y su conservación, pp. 37-45. Fundación de Historia Natural Félix de Azara, Universidad Maimónides, Buenos Aires.

BACIGAlUPO, N. M.; G. DELUCCHI; J. A. HURRELL \& N. M. TUR. 2008. En: Flora rioplatense: sistemática, ecología y etnobotánica de las plantas vasculares rioplatenses. Parte 3. Vol. 1. Monocotiledóneas (J. A. Hurrell, dir.). L.O.L.A. (Literature of Latin America), Buenos Aires. 336 pp.

BARBOZA, G. E.; J. J. CANTERO; C. O. NÚÑEZ \& L. ARIZA ESPINAR (Eds.). 2006. Flora medicinal de la provincia de Córdoba (Argentina). Museo Botánico de Córdoba. 1252 pp.

CABRERA, A. L. 1949. Las comunidades vegetales de los alrededores de La Plata, provincia de Buenos Aires, República Argentina. Lilloa 20: 269-274.

DE PAULA F.; M. LOCKS; M. BELTRÃO \& M. AMARAL. 2002. Estudo preliminar etnobotânico de plantas de uso medicinal na Região arqueológica de Central-Bahia, Brasil. Tercer Congreso Virtual de Antropología y Arqueología. Naya. (http://www. naya.org.ar/congreso2002/ponencias/martha_locks. htm).

HERNÁNDEZ M. P.; M. N. COLARES \& S. M. CIVITELLA. 2009. Plantas utilizadas en medicina popular en un sector del Partido de Berisso, Buenos Aires, Argentina. (BLACPMA) Bol Latinoam Caribe Plant Medic Aromat 8(4): 435-444.

; S. M. CIVITELlA \& V. G. ROSATO. 2010. Uso medicinal popular de plantas y líquenes de la Isla Paulino, provincia de Buenos Aires, Argentina. (BLACPMA) Bol Latinoam Caribe Plant Medic 
Aromat 9(4): 258-269.

HILGERT, N. I.; M. L. HIGUERA\& M. J. KRISTENSEN. 2010. La medicina herbolaria en el contexto urbano. Estudio de caso en un barrio de la ciudad de Tandil, Argentina. (BLACPMA) Bol Latinoam Caribe Plant Medic Aromat 9(3): 177-190.

HURRELL, J. A.; G. DELUCCHI; M. N. CORREA; M. I. SÁNCHEZ; G. ROITMAN; F. B. COSTANTINO; E. A. ULIBARRI; E. R. GUAGLIANONE \& N. M. TUR. 2009. Flora rioplatense: sistemática, ecología y etnobotánica de las plantas vasculares rioplatenses (J. A. Hurrell, dir.). Parte 3. Vol. 4. Monocotiledóneas. L.O.L.A. (Literature of Latin America), Buenos Aires. 424 pp.

; E. A. ULIBARRI; J. P. PUENTES; F. BUET COSTANTINO; P. M. ARENAS \& M. L. POCHETTINO. 2011. Leguminosas medicinales y alimenticias utilizadas en la conurbación Buenos Aires-La Plata, Argentina. (BLACPMA) Bol Latinoam Caribe Plant Medic Aromat 10 (5): 443 -455 .

KELLER, H. A. 2008. Las plantas usadas en la construcción y el acondicionamiento de las viviendas y templos guaraníes en Misiones, Argentina. Bonplandia 17(1): 65-81.

— \& H. F. ROMERO. 2006. Plantas medicinales de la Reserva de Biósfera Yabotí (Misiones, Argentina). Bonplandia 15(3-4): 125-141.

LAHITTE, H. B. \& J. A. HURRELL (dir.). 1995-1996. Las plantas de la medicina popular de la isla Martín García (nativas y naturalizadas). Comisión de Investigaciones Científicas, Ministerio de la Producción de la Provincia de Buenos Aires. 246 pp.

—; J. A. HURRELL; M. L. BELGRANO; L. S. JANKOWSKI; K. MEHLTRETER; M. P. HALOUA \& G. CANDA. 1997. Plantas de la costa. L.O.L.A. Buenos Aires. 200 pp.

—_ J. A. HURRELL; M. L. BELGRANO; L. S. JANKOWSKI; M. P. HALOUA \& K. MEHLTRETER. 1998. Plantas medicinales rioplatenses. L.O.L.A. Buenos Aires. 240 pp.

MANDRILE, E. L. 2003. Farmacognosia: plantas medicinales que se dispensan en Argentina. Colegio de Farmacéuticos de la provincia de Buenos Aires. $289 \mathrm{pp}$.

MARTÍNEZ CROVETTO, R. 1964. “Estudios etnobotánicos I. Nombres de plantas y su utilidad, según los indios tobas del este del Chaco. Bonplandia 1(4): 279-333.

. 1965. "Estudios etnobotánicos II. Nombres de plantas y su utilidad según los indios vilelas del Chaco. Bonplandia 2(1): 123.

- 1981. Plantas utilizadas en medicina en el noroeste de Corrientes. Fundación Miguel Lillo, Tucumán. $139 \mathrm{pp}$.

MARTÍNEZ, M. R. \& M. L. POCHETTINO. 1992. The 'farmacia casera' (household pharmacy): a source of ethnopharmacobotanical information. Fitoterapia 63(3): 209-216.

MARZOCCA, A. 1997. Vademécum de malezas medicinales de la Argentina. Indígenas y exóticas. Hemisferio Sur, Buenos Aires. 363 pp.

MICHELLOD, O. E. 2000. La identidad del paisaje urbano a través de la memoria. Berisso, Argentina. Al Márgen. 300 pp.

2001. Paisaje: reflexiones. Al Márgen. 329 pp.

POCHETTINO, M. L.; M. R. MARTÍNEZ; B. ITTEN \& M. ZUCARO. 1997. El uso de plantas medicinales en la atención primaria de la salud: estudio etnobotánico en una población urbana (Pdo. La Plata, Prov. Buenos Aires, Argentina). Parodiana 10(1-2): 141-152.

-; P. ARENAS; D. SÁNCHEZ \& R. CORREA. 2008. Conocimiento botánico tradicional, circulación comercial y consumo de plantas medicinales en un área urbana de Argentina. (BLACPMA). Bol Latinoam Caribe Plant Medic Aromat 7(3): 141-148.

SANUCCI, L. E. M. 1972. Berisso, un reflejo de la evolución Argentina. Plan regulador de la Ciudad de Berisso, Buenos Aires. 300 pp.

TOURSARKISSIAN, M. 1980. Plantas medicinales de la Argentina. Hemisferio Sur, Buenos Aires. 178 pp.

VALLA, J. J.; L. S. JANKOWSKI; D. BAZZANO \& A. J. HERNÁNDEZ. 1999. En: Lahitte H. B. \& J. A. Hurrell (eds.), Biota rioplatense. IV. Árboles urbanos. L.O.L.A. (Literature of Latin America), Buenos Aires. 320 pp.

ZULOAGA, F. O.; O. MORRONE \& M. J. BELGRANO. 2008. Catálogo de las Plantas vasculares del Cono Sur (Argentina, Sur de Brasil, Chile, Paraguay y Uruguay). Monogr. Syst. Bot. Missouri Bot. Gard. 107: 1-3348. Edición actualizada "on line", consultada abril 2011 (www.darwin.edu.ar/).

Original recibido el 13 de junio de 2011; aceptado el 25 de noviembre de 2011. 